\title{
Frontal Alopecia after Repeated Botulinum Toxin Type A Injections for Forehead Wrinkles: An Underestimated Entity?
}

\author{
Antonino Di Pietro ${ }^{a}$ Bianca Maria Piraccini ${ }^{b}$ \\ astituto Dermoclinico Vita Cutis, San Donato Hospital Group, Milan, and bivision of Dermatology, \\ Department of Specialized, Diagnostic and Experimental Medicine, University of Bologna, Bologna, Italy
}

\section{Key Words}

Alopecia · Botulinum toxin · Wrinkles · Frontal alopecia

\begin{abstract}
Background: Injections of botulinum toxin type $A$ in the forehead have never been reported to cause hair side effects. Objective: The aim of this paper is to report a new type of alopecia, which we have seen in women undergoing periodic injections of botulinum toxin type $A$ for forehead wrinkles, and to differentiate it from other types of hair loss. Methods: We conducted an observational study on $5 \mathrm{fe}-$ males recruited from a private and an institutional practice who complained of progressive recession of the hairline after periodic injections of botulinum toxin type $A$ in the forehead. Results: Alopecia of the frontal hairline was evident in all 5 patients, with absence of skin atrophy or scarring and progressive hair miniaturization at trichoscopy. Conclusion: Dermatologists should be aware of the possible occurrence of frontal alopecia after repeated injections of botulinum toxin type $\mathrm{A}$ for forehead wrinkles.
\end{abstract}

(C) 2016 S. Karger AG, Base

\section{Introduction}

Injections of botulinum toxin type A (BTXA) are widely performed in cosmetology to decrease facial and neck wrinkles, and in the last 10 years, the use of this technique has become more and more common worldwide, even in young adults [1], with a high satisfaction rate [2]. Side effects are generally mild and cause little discomfort; excessive dose or spread of the toxin to nearby muscles cause temporary drooping. More rarely, transient headache, nausea, or flu-like symptoms have been reported. Skin and hair changes have not been included as side effects so far.

We report the cases of 5 females who developed regression of the frontal hairline after several treatments with BTXA for forehead wrinkles.

The patients had a mean age of 52.7 years (range 4558) and had started to undergo periodic BTXA injection for forehead wrinkles $1-10$ years ago (mean 2.6$)$. The frequency of injections ranged from 4 to 6 months, and the sites injected in addition to the forehead included the periorbital and perioral areas in 3 cases, and the periorbital area alone in 1 patient. We could not assess the dose of BTXA utilized in each case, as all patients performed the procedures in other dermatology/plastic surgery settings.

\section{KARGER}

E-Mail karger@karger.com www.karger.com/sad
(2) 2016 S. Karger AG, Basel

2296-9195/16/0022-0067\$39.50/0
Prof. Bianca Maria Piraccini

Division of Dermatology, Department of Specialized, Diagnostic and Experimental Medicine, University of Bologna

Via Massarenti, 1, IT-40138 Bologna (Italy)

E-Mail biancamaria.piraccini@unibo.it 


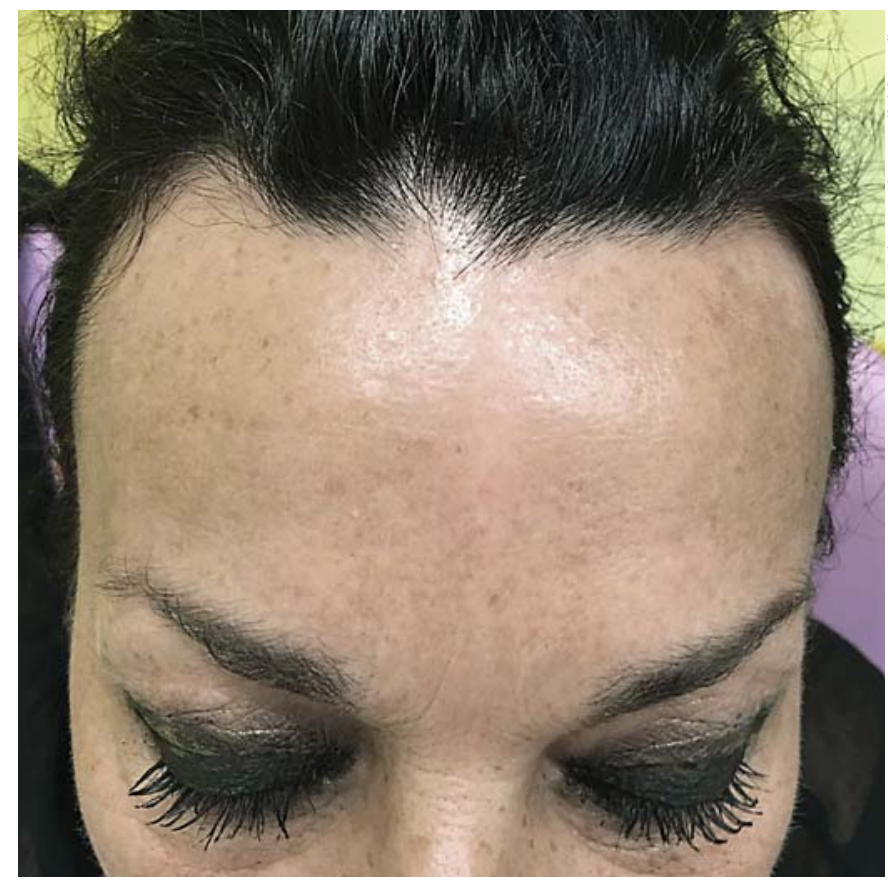

Fig. 1. Evident regression of the frontal hairline in a 52-year-old female undergoing periodic forehead BTXA injections for wrinkles.

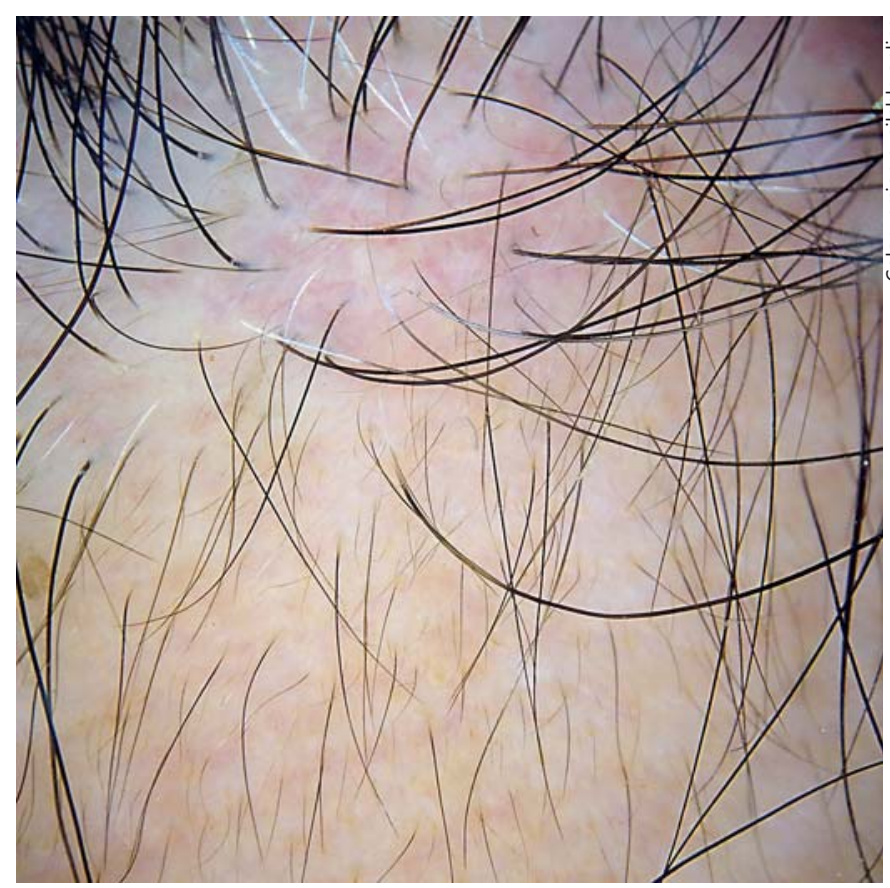

Fig. 2. Trichoscopy of the frontal hairline showing skin with signs of photoaging and evident follicular ostia with vellus, intermediate and terminal hair.
All women had noticed a gradual regression of the frontal hairline that made the forehead higher than before (fig. 1). Although no patient could provide a picture showing the features of the hairline before the hair loss, all were very aware of their physical features and extremely conscious of this progressive alopecia. The mean distance between the hairline and the glabella was $>6 \mathrm{~cm}$.

In all cases, clinical and trichoscopic examination of the forehead showed a normal skin with signs of photoaging, no signs of atrophy or scarring and visible follicular ostia with vellus and intermediate hair along the hairline (fig. 2). Neither inflammatory signs nor follicular hyperkeratosis were detectable.

Examination of the scalp and of other body areas, including eyebrows and eyelids, did not show any sign of hair loss except for a mild form of female androgenetic alopecia in a 53-year-old patient. The patients were otherwise healthy, and 3 of them were in menopause ( 1 patient underwent hormonal therapy).

\section{Discussion}

We hypothesized that this form of alopecia can be related to BTXA injection and coined the name 'botulininduced frontal alopecia (BIFA)' in order to diffuse the knowledge and the study of this new form of hair loss.

Unfortunately, none of our patients allowed us to perform a biopsy form the forehead for histopathological study, and we therefore cannot assess the pathological aspects of BIFA. Trichoscopy does not show scarring or atrophy, and together with the clinical features, it permits to differentiate BIFA from frontal fibrosing alopecia (FFA). In FFA, which is considered a variant of lichen planopilaris, there is a progressive regression of the frontal hairline, often associated with involvement of the parietal hair margin and with alopecia of the eyebrows; the alopecic skin typically lacks signs of photoaging and of vellus and intermediate hair, with loss of follicular ostia [3]. The terminal hairs at the hairline often show mild follicular hyperkeratosis and perifollicular erythema. BIFA, on the other hand, is not characterized by scarring but rather by a gradual miniaturization of the hair follicles of the frontal hairline, making hairs decrease in size and turning into vellus hair. In this way, the clinical and trichoscopy features are more similar to those seen in androgenetic alopecia of the temporal hairline [4].

Similar to FFA, however, BIFA seems to increase in prevalence with time, as 4 of the 5 cases reported here have been seen in the last 4 months. This is probably due
Di Pietro/Piraccini 
to the increased worldwide frequency of the procedure of BTXA injection for wrinkles.

We could not find any report of botulin-related hair loss similar to that of our patients in the literature. Wabbels and Stanzel [5] reported a patient with eyebrow alopecia following repeated injections of BTXA for blepharospasm, which turned out to be alopecia areata associated with scalp patches. Paradoxically, there are 2 studies that evaluated the effectiveness of BTXA injections for the treatment of alopecia areata [6] and androgenetic alopecia [7], both with poor results. While in androgenetic alopecia the rationale for the use of BTXA is its effect to loose the scalp, allowing a better vascularization that could promote hair follicle growth, in the treatment of alopecia areata, BTXA injections have been tried with the idea of inducing changes in neurotransmitter levels, influencing the production of cytokines responsible for hair growth arrest.

BTXA is a potent neurotoxin that enters the presynaptic bulb of cholinergic neurons and prevents acetylcholine release into the synaptic cleft, blocking both somatic and autonomic innervation. BTXA activity of suppressing muscle contractility is utilized for the treatment of several neurogenic dysfunctions characterized by excessive or inappropriate muscle contractions, such as strabismus, blepharospasm or muscular dystonias [8], as well as for cosmetic purposes to improve facial lines [9]. Other important in-label and off-label uses of BTXA in dermatology are severe primary axillary hyperhidrosis and palmar hyperhidrosis, respectively, where the toxin is injected intradermally [10]. Side effects are usually the result of inexpert injection and can be avoided if the physician injecting BTXA is properly trained.
Alopecia is never mentioned as a possible side effect of BTXA injections, and its occurrence is difficult to explain. Possible explanations include changes in the levels of molecules that may be involved in regulating the hair cycle, i.e. substance $\mathrm{P}$ and calcitonin gene-related peptide and other signaling molecules and receptors [11], and/or decreased neurological stimulation of hair follicle function due to blockage of the function of autosomic fibers [12].

We present this new form of alopecia, which we named BIFA, in order to raise awareness of this possible side effect of forehead BTXA injections for wrinkles.

\section{Conclusions}

Dermatologists should be aware of frontal alopecia as a possible side effect of BTXA injections and carefully evaluate patients if they realize a change in their frontal hairline. Collection of more cases and histopathological/ immunohistochemical studies will be necessary to understand the real prevalence and etiopathogenesis of this entity.

\section{Statement of Ethics}

Subjects have given their informed consent.

\section{Disclosure Statement}

The authors declare no conflicts of interest.

\section{References}

1 Carruthers A, Sadick N, Brandt F, et al: Evolution of facial aesthetic treatment over five or more years: a retrospective cross-sectional analysis of continuous onabotulinumtoxin $\mathrm{A}$ treatment. Dermatol Surg 2015;41:693-701.

2 Rivers JK, Bertucci V, McGillivray W, et al: Subject satisfaction with onabotulinumtoxinA treatment of glabellar and lateral canthal lines using a new patient-reported outcome measure. Dermatol Surg 2015;41:950-959.

3 MacDonald A, Clark C, Holmes S: Frontal fibrosing alopecia: a review of 60 cases. J Am Acad Dermatol 2012;67:955-961.
4 Rudnicka L, Olszewska M, Rakowska A, Slowinska M: Trichoscopy update 2011. J Dermatol Case Rep 2011;5:82-88.

5 Wabbels B, Stanzel BV: Unilateral loss of eyebrows and repeated botulinum toxin injections: questionable correlation in essential blepharospasm. Ophthalmologe 2015;112: 174-176.

6 Cho HR, Lew BL, Lew H, Sim WY: Treatment effects of intradermal botulinum toxin type A injection on alopecia areata. Dermatol Surg 2010;36(suppl 4):2175-2181.

7 Freund BJ, Schwartz M: Treatment of male pattern baldness with botulinum toxin: a pilot study. Plast Reconstr Surg 2010;126:246e248e.
8 Carruthers J, Carruthers A: Botox: beyond wrinkles. Clin Dermatol 2004;22:89-93.

9 Lowe NJ, Lowe P: Botulinum toxins for facial lines: a concise review. Dermatol Ther (Heidelb) 2012;2:14.

10 Hosp C, Naumann MK, Hamm H: Botulinum toxin treatment of autonomic disorders: focal hyperhidrosis and sialorrhea. Semin Neurol 2016;36:20-28

11 Kim DW, Lee SK, Ahnn J: Botulinum toxin as a pain killer: players and actions in antinociception. Toxins (Basel) 2015;7:2435-2453.

12 Paus R: Frontiers in the (neuro-)endocrine controls of hair growth. J Investig Dermatol Symp Proc 2007;12:20-22. 